\title{
Konstruierte Naturen und ihre Erforschung
}

Die Natur führt in der Geographie ein schillerndes Dasein. Für die inhaltliche Einteilung wie für die formale Verfaßtheit der Disziplin ist der Begriff grundlegend. Zugleich scheint die Beschäftigung mit den verschiedenen Konzeptionen von Natur seit langem fast verschwunden, so daß sich in geographischen Texten nur noch «geisterhafte Spuren eines Konzepts» verfolgen lassen, wie jüngst Kenneth OLWIG (1996) schrieb.

Als Schlüsselkategorie der Geographie wurde die Natur historisch mit dem Naturdeterminismus und der ganzheitlichen «Landschaft» verabschiedet (vgl. HARD 1983: 162f.). Im scheinbar neutraleren Begriff des Raumes wurden jedoch einige Probleme, die mit dem Naturbegriff verbunden waren, weitergeschleppt: die Vorstellung von dem immer präsenten und letztgründigen Container, der alles Soziale umschließt, aber auch die passiven Eigenschaften einer Matrix und Ressource. Vor allem läßt sich auch der Raumkausalismus, dessen Relikte in der deutschsprachigen Humangeographie durch konsequente Einfuihrung soziologischer Theorie eben erst getilgt werden, zu guten Teilen als Verlängerung des traditionellen Natur- und Landschaftsgedankens identifizieren (WERLEN 1997). Schon deshalb wäre es verfehlt, heute dort anknüpfen zu wollen, wo die Geographie die Kategorie Natur weitgehend aufgegeben hat. Jedoch haben andere Disziplinen die Kategorie in den letzten fünfzehn Jahren erheblich weitergedacht, und so kehrt sie nun - wie noch zu zeigen bleibt - aufgefrischt hier und dort in die Geographie zurück. Dabei wird immer häufiger gerade die Trennung von Natur und Gesellschaft neu in Frage gestellt, die in der Geographie institutionalisiert ist - in Teilen der humangeographischen Diskussion aber begrifflich noch kaum erreicht wurde und in der physischen Geographie unhinterfragt bleibt.

Die «konstruierten Naturen» stehen demnach zwischen zwei Feldern: Während die unendliche Geschichte des Naturdeterminismus und der ganzheitlichen Landschaft noch hier und da aufscheint, zeichnet sich am Horizont schon ein neues Feld ab, in dem sich soziale Objekte und Quasisubjekte tummeln und wo sich die traditionellen Grenzen von sozialer und natürlicher Sphäre neu zu verflüssigen scheinen (LATOUR 1995, KNORRCETINA 1997). Jenes Zwischenfeld, die Debatte um «konstruierte Naturen», soll im Folgenden umrissen werden. Anschließend werde ich der Frage nachgehen, welche Forschungsperspektiven für die Geographie sich darin eröffnen, und dies an einem jüngeren Beitrag über die Waldkonflikte in British Columbia illustrieren. Den
Schluß bildet ein Plädoyer für die theoretische Erneuerung der Beschäftigung mit den Grenzen von Natur und Kultur in der Humangeographie entlang aktueller Konflikte.

\section{Inwiefern konstruiert?}

Zunächst einmal muß die Rede von den «konstruierten Naturen» näher bestimmt werden. Ist damit gemeint, daß es gar keine wirkliche Natur mehr gibt? Dreißig Jahre nach BERGER und LUCKMANNS berühmtem Buch über die gesellschaftliche Konstruktion der Wirklichkeit (1969) scheint heute alles auf irgendeine Art «konstruiert», und so taucht auch die Natur als Konstrukt in so verschiedenen Zusammenhängen und Disziplinen auf, daß eine kurze Sortierung der wichtigsten Verwendungsweisen und ihrer theoretischen Bezüge nützlich erscheint.

Alle Perspektiven, in denen von einer konstruierten Natur die Rede ist, eint zumindest, daß sie den Zugang zu dem, was wir ohne weiteres Nachdenken für Natur (im Gegensatz zu Kultur oder Gesellschaft) halten, für problematisch oder prekär erachten. Jede Wahrnehmung, Überlegung, Äußerung und Handlung in bezug auf diese Natur, ob in der Wissenschaft oder anderswo, sei vermittelt, sie treffe immer schon auf eine «Konstruktion». Zugespitzt in den Worten von HARAWAY (1992: 296): «Die Natur kann nicht vor ihrer Konstruktion existieren.»

Die unterschiedlichen Denkrichtungen betonen jedoch ganz verschiedene Konstruktionsweisen und nehmen dabei auf eine Vielfalt theoretischer Ansätze Bezug, die sich wohlgemerkt oft nicht primär auf den Betrachtungsgegenstand Natur beziehen. Vor allem zu Verständigungszwecken und ohne Anspruch auf Vollständigkeit schlage ich vor, wenigstens vier Perspektiven zu unterscheiden (vgl. Abbildung 1):

1. Als die erkannte Natur lassen sich zwei im engeren Sinne konstruktivistische Perspektiven zusammenfassen, die beide am Erkenntnisproze $ß$ ansetzen, seit den achtziger Jahren aber über Erkenntnistheorie und Wissenschaftsforschung hinaus von Einfluß waren.

Michael Flitner, Dr., DFG Graduiertenkolleg «Sozioökonomie der Waldnutzung in den Tropen", Albert-Ludwigs-Universität, Freiburg i. Br. 
Erstens ist dies der kognitionsbiologische Konstruktivismus, in dem die Art der Informationsverarbeitung des Gehirns zum Ausgangspunkt weitreichender Überlegungen gemacht worden ist, wonach es grundsätzlich keine «naturgetreue» Abbildung der Wirklichkeit geben könne, sondern die Beobachtungen ein Konstrukt des Beobachtenden seien (MATURANA \& VARELA 1987). Das die Welt konstruierende Gehirn hat besonders in systemtheoretischen Ansätzen Widerhall gefunden, prominent wurde dieses «selbstbeobachtende System» etwa in der Luhmannschen Systemtheorie.

Zweitens stellt auch der sogenannte Laborkonstruktivismus die Erkenntnis ins Zentrum, jedoch steht dabei nicht mehr die Leistung des Gehirns im Vordergrund, sondern die «Herstellung» (natur)wissenschaftlicher Fakten im Labor (LATOUR \& WOOLGAR 1979, KNORR-CETINA 1981). In diesem stark von der Ethnomethodologie inspirierten Forschungsprogramm wird vor allem die erkenntnispraktische Kontingenz allen wissenschaftlichen Wissens betont und in einer Mikroperspektive erforscht. Die Natur, so heißt es, gebe uns keine «Daten», unsere Befunde, gerade über die Mikrostrukturen der belebten und unbelebten Natur, würden vielmehr in der Wissenschaft "gemacht» und verhandelt.

2. Die bedeutende oder kulturelle Natur könnte dagegen die Ansätze bezeichnen, die im weitesten Sinne kulturtheoretisch inspiriert sind. Besonders die Ethnologie befaßt sich in vielfacher Weise mit der Repräsentation und (Be-)Deutung von Natur, jedoch nicht als eine Mikrokonstruktion des Gehirns oder der Wissenschaft, sondern als geschichtlich-kulturelles Produkt, in Form von Klassifikationen, Symbolen und Mythen (ELLEN \& FUKUI 1996). Dabei geht es sowohl um die «kognitive Organisation» von Individuen und Gruppen im Hinblick auf ihre Wahrnehmung, Abgrenzung und Kategorisierung von Natur als auch um deren normative und symbolische Funktion (EDER 1988). Die theoretischen Einflüsse sind zahlreich und kaum zu vereinheitlichen, wesentliche Impulse kommen unter anderem aus der Nachfolge des französischen Strukturalismus (DESCOLA 1996), der (Kultur-)Soziologie (EDER 1988), der feministischen Philosophie (BUTLER 1995) und neuerdings auch aus den literaturwissenschaftlich beeinflußten cultural studies of science (HARAWAY 1992, 1997). Schließlich lassen sich in dieses Feld auch Ansätze rechnen, die sich in einer symboltheoretischen Perspektive mit der bildlichen Repräsentation von Natur befassen wie die in der Tradition der kritischen Theorie stehende «politische Ikonographie» (WARNKE 1992). Der Blick richtet sich in vielen dieser Ansätze keineswegs mehr vorzugsweise auf «fremde», nichtwestliche Kulturen. Grundlegende Naturmythen und darauf bezogene Normen lassen sich ebenso in den Gesellschaften aufspüren, die sich für aufgeklärt erachten (THOMPSON U. A. 1990, CRONON 1995).
3. Die produzierte Natur ist die durch Arbeit transformierte Natur, die Natur der Technik und der Warenproduktion, der Pflanzungen und Wüstungen, der Forste und der Gentechnik. Unter diesem Schlagwort lassen sich die Perspektiven gruppieren, die die materielle Dimension menschlicher Naturaneignung und -gestaltung ins Zentrum stellen. Theoretisch ist dieser Blick vor allem von marxistischen und regulationstheoretischen Einflüssen geprägt (SMITH 1984, HARVEY 1996, GÖRG 1998), aber auch Teile der environmental history lassen sich hier zuordnen (vgl. WILLIAMS 1994). Nur wenige Autoren gehen dabei so weit, die Natur heute ganz als eine «menschliche Geschichte» zu konzipieren, in der es überhaupt keinen Naturzustand jenseits des Sozialen mehr geben kann (MOSCOVICI 1982). Insofern die normative und symbolische Dimension in der Perspektive "produzierte Natur» stark zurücktreten, läßt sich wohl nur noch in einem sehr weiten Sinne von konstruierter Natur sprechen. Wie bei der «kulturellen Natur» fließt jedoch auch hier häufig die Vorstellung einer sekundären Naturalisierung ein, wie sie der breitere Sozialkonstruktivismus im Sinne von BERGER \& LUCKMANN (1969) im Hinblick auf gesellschaftliche «Tatsachen» formuliert hat. Die angeeignete, sozial gewordene Natur wird demnach sekundär objektiviert durch die Symbolisierung und die Versprachlichung der Erfahrung sowie durch die Routinisierung und Habitualisierung von Handlungen. Die produzierte Natur wird so zu einem Teil der sozialen «zweiten Natur».

4. Die simulierte Natur schließlich wird von Tamagotchis und computeranimierten Avataren bevölkert; die Videoclips aus der Warenwelt gehören ihr ebenso an wie die Simulationen der Klimaforscher. Die Rede von einer simulierten oder virtuellen Natur, in der die Realität selbst nur noch als Illusion und Verführung existiert, ist mittlerweile über Endzeitessayistik und Journalismus schon so tief in das Alltagsbewußtsein vorgedrungen, daß sie ihren philosophischen Schrecken weitgehend verloren hat und kaum noch mehr assoziieren läßt als die (auf Dauer doch enttäuschenden) SimEarth- und SimLife-Spiele auf dem Homecomputer. Die theoretische Beschäftigung mit diesen künstlichen Welten ist maßgeblich von der Medientheorie, der Semiotik und der Informationswissenschaft inspiriert (BAUDRILLARD 1978, ECO 1987, McKIBBEN 1990, HAYLES 1995). In der extremen Variante wird jede Unterscheidung zwischen Konstrukt, Realität und Schein hinfällig, und am Ende bleiben nur noch «Inszenierungen zur Wiederbelebung der Fiktion des Realen» (WELSCH 1993: 150). In der bescheideneren Variante entsteht immerhin eine "dritte Natur» (WARK 1994), nämlich eine Informationslandschaft aus Datenbanken und -autobahnen, die gegenüber der bereits sozial konstruierten «zweiten Natur» zunehmend Eigenständigkeit erlangt. 


\begin{tabular}{|c|c|}
\hline $\begin{array}{c}\text { Erkannte Natur } \\
\text { (Gehirn /Labor) }\end{array}$ & $\begin{array}{c}\text { Kulturelle Natur } \\
\text { (Klassifikationen / } \\
\text { Symbole / Mythen / } \\
\text { Normen) }\end{array}$ \\
\hline $\begin{array}{c}\text { Simulierte Natur } \\
\text { (Zeichen / Daten / } \\
\text { Medien) }\end{array}$ & $\begin{array}{c}\text { Produzierte Natur } \\
\text { (Technik/ } \\
\text { Warenproduktion) }\end{array}$ \\
\hline
\end{tabular}

Abb. 1: Konstruierte Naturen

Diesen vier Typen «konstruierter Natur» lassen sich nun keineswegs alle Forschungsperspektiven eindeutig zuordnen. Die verschiedenen Blickwinkel befinden sich auf unterschiedlichen theoretischen Ebenen, sie überschneiden sich vielfach, schließen sich von ihren Grundannahmen her zum Teil aber auch aus. Eine allumfassende Ordnung oder gar eine Metatheorie soll und kann in einem solchen Schema demnach nicht erreicht werden. Die Untergliederung in die genannten vier Naturen stellt nicht mehr als einen ersten Versuch dar, wichtige Pole und theoretische Einflüsse in der Debatte um «konstruierte Naturen» sichtbar zu machen.

Zwei Punkte sollen in Ergänzung dieses Klärungsversuchs noch kurz angesprochen werden, weil sie Anlaß zu mancher Verwirrung geben. Erstens: Die pauschale Rede von einer «diskursiven Wende» oder einer «linguistischen Wende» (GERBER 1996) ist im Zusammenhang mit den konstruierten Naturen wenig hilfreich. Zwischen den einzelnen Perspektiven gibt es so grundlegende und weitreichende Differenzen, daß ein Begriff wie "diskursiv» eher Verwirrung stiftet, wenn darin Strukturalismus, Sprachpragmatik und Soziolinguistik mit einer Prise Foucault unbekümmert verrührt werden. Irreführend ist die Annahme einer Hinwendung zum «Sprachlichen» jedenfalls bei Autoren wie Foucault, die im Blick auf den Strukturalismus die Privilegierung der Sprache und der Zeichensysteme gerade in Frage stellen (vgl. SPIVAK 1996: 302). So würden Ansätze, die in dem hier behandelten Kontext auf den Foucaultschen Diskursbegriff zurückgehen, Elemente aus den Feldern zusammendenken, die oben als die erkannte, die kulturelle und die produzierte Natur idealtypisch getrennt wurden, und zwar auf eine Art, die gerade die Unauflöslichkeit der Verbindung von Macht, Erkenntnis und materialer Naturaneignung ins Zentrum stellt. Eine etwas genauere Einordnung ist also jedenfalls dann nötig, wenn es um die theoretischen und methodologischen Implikationen der verschiedenen Perspektiven geht.

Zweitens bleibt die eingangs aufgeworfene Frage, welchen Realitätsgehalt Natur in den verschiedenen Perspektiven denn nun hat, also ob es eigentlich im ontologischen Sinne eine "echte Natur» hinter oder neben den verschiedenen konstruierten Naturen gibt. An dieser Frage entzünden sich, kaum überraschend, im- mer neue Polemiken um den Sinn «antirealistischer» Positionen und die Postmoderne im allgemeinen (vgl. SOKAL 1996). Sie erweist sich bei genauerem Hinsehen jedoch als erstaunlich wenig trennscharf und in vielen Ansätzen nicht einmal direkt von Belang. Die konstruktivistische Perspektive im engeren Sinne - hier vor allem: die erkannte Natur - macht in den meisten Fällen keine Aussagen darüber, ob es Natur gibt, sondern nur darüber, ob und wie wir sie (oder überhaupt eine Wirklichkeit) erkennen können. Und in den Ansätzen, die hier als kulturelle und als produzierte Natur bezeichnet wurden, halten sich in mehr oder minder versteckter Form noch mancherlei realistisch-naturalistische Grundpositionen. Das heißt: Wenn von konstruierten Naturen gesprochen und geschrieben wird, ist die Frage nach dem ontologischen Status von Natur oder dem materiellen Gehalt der Konstruktion «Natur» noch nicht zwingend beantwortet. Vor allem kann die Konstruktion ein rein erkenntnistheoretisches oder methodologisches Postulat sein.

Deshalb eignet sich dieses Kriterium auch nur bedingt als Trennlinie für die verschiedenen Blickwinkel, wie ich sie oben zu ordnen versucht habe. Allerdings bleibt die Frage nach der Materialität virulent, ja man kann sogar sagen: aus ihr bezieht die Rede von der konstruierten Natur ihren hauptsächlichen Reiz. Die vollständige Auflösung aller Natur nach der sozialen/kulturellen Seite hin ist theoretisch nur wenig befriedigender als der Naturalismus, der zurückbleibt, wenn man - wie in der Umweltdiskussion weithin üblich - den Konstruktionsaspekt schlicht «vor die Klammer zieht» (VAN DEN DAELE 1996: 421).

\section{Die Erforschung konstruierter Naturen in der Geographie}

Wenn die Ausgangspunkte und theoretischen Quellen der Rede von konstruierten Naturen so vielfältig sind, läßt sich konsequenterweise auch kein schlüssiges Forschungsprogramm und erst recht keine einheitliche Methodologie entwickeln, wie diese Naturen aus der Perspektive der Geographie (oder sonst einer Wissenschaft) zu bearbeiten wären. Zumindest bleibt aber festzustellen, in welcher Weise die umrissene Debatte hier bisher Wirkung gezeigt hat. Auf dieser Grundlage werden zugleich die Leerstellen, die Nichtbeschäftigung mit bestimmten Problemen deutlich, und so lassen sich zumindest einige inhaltliche Gebiete und theoretischmethodische Fragestellungen andeuten, deren Bearbeitung lohnend erscheint.

Die Quellen, denen die Natur als Kategorie ihr Wiedererwachen in der Geographie verdankt, sind relativ klar zu benennen. Zunächst einmal läßt sich festhalten, daß das neue Interesse zwar mit der Konjunktur ökologischer Probleme zusammenhängt, jedoch zum größten Teil nicht aus der konkreten Erforschung naturwissenschaftlich bestimmter Problemlagen resultiert. Vielmehr kommt diese Bewegung (auch) in der Geographie von 
der sozial- und kulturwissenschaftlichen Seite her, die im letzten Jahrzehnt verstärkt wieder Zugang sucht zu einem Feld, durch dessen Ausschluß sie sich selbst definieren zu müssen glaubte - vor allem in theoretischmethodischer Hinsicht, aber von Ausnahmen abgesehen auch was den Gegenstand anbelangt. Ähnlich wie die Soziologie hat auch die Humangeographie nur zögerlich und vereinzelt innovativ auf den politischen «Ökoboom» der siebziger und achziger Jahre reagiert (vgl. ZIMMERER 1996). Weder der Ökosystemansatz noch die Ausläufer einer kulturhistorischen Ökologie in der Tradition von C.O. Sauer konnten verhindern, daß insgesamt «die Geographie auf ihrem ureigensten Gebiet versagt» (BAHRENBERG 1994: 57). Jedenfalls hat sie bisher kaum eine herausragende Rolle gespielt, das erneuerte Interesse am Mensch-Natur-Verhältnis derart interdisziplinär zu erforschen, wie es die Fachstruktur nahezulegen scheint.

Das Bedürfnis, in der Auseinandersetzung mit der Politisierung ökologischer Fragen den Naturbegriff theoretisch zu erneuern, ist in der Humangeographie am intensivsten in der angelsächsischen Literatur über die «produzierte Natur» zu erkennen. Marx' berühmter «Stoffwechsel» zwischen Mensch und Natur, der in der Vergangenheit (ontologisch und epistemologisch) ganz überwiegend realistisch, streng anthropozentrisch und meist technokratisch verstanden wurde, wird seit einigen Jahren kritisch überdacht. Dabei kommen vor allem Elemente aus den Feldern zum Tragen, die hier als die kulturelle und die erkannte Natur bezeichnet wurden. Während SMITH (1984) und FITZSIMMONS (1989) noch gegen ein naturalistisches Verständnis von «erster Natur» anschreiben, werden mit den Arbeiten von MOORE (1993) zu den Landkonflikten in Zimbabwe und ESCOBAR (1996) zu den neuen Formen «ökologischen Kapitals» in Kolumbien Elemente aus dem Poststrukturalismus aufgenommen; die theoretischen Überblicke bei CASTREE (1995) Sowie CASTREE \& BRAUN (1998) beziehen schließlich auch die neuere Wissenssoziologie systematisch mit ein.

In diesen Arbeiten formiert sich zugleich ein wichtiges Einflußmoment auf das gesamte Feld der (neuen) political ecology (BLAIKIE 1995, BRYANT \& BAILEY 1997). Die Forschungsrichtung, die sich im letzten Jahrzehnt unter diesem theoretisch noch recht vagen Konzept versammelt hat, könnte seit langem der erste Ansatz zur Analyse der gesellschaftlichen Naturverhältnisse sein, der maßgeblich von der (Human-)Geographie geprägt wird. In erster Linie mag dies dem starken Bezug zu entwicklungspolitischen Fragen und der Vorliebe für regionenbezogene empirische Fallstudien geschuldet sein. In der Analyse von Umweltproblemen in Afrika (WATTS 1989 über Ernährungssicherung in Nigeria), Südamerika (HECHT \& COCKBURN 1989 über Ressourcenkonflikte in Amazonien) und Asien (PELUSo 1992 über die indonesische Waldpolitik) ergeben sich Verbindungen zu Sichtweisen der Ethnologie, der Kultursoziologie und der Politikwissenschaft, die die niederliegenden Reste der radical geography und der kulturhistorischen Ökologie mit den obengenannten theoretischen Einflüssen aufgefrischt haben (vgl. auch den Sammelband von PEET \& WATTS 1996).

Während die Beschäftigung mit der produzierten Natur (neben der Akteursorientierung und der Mehrebenenanalyse) bei aller Uneinheitlichkeit zu den Ausgangspunkten der political ecology gerechnet werden dürfte, steckt die Auseinandersetzung mit diskursanalytischen und kultursoziologischen Theorien und Methoden insgesamt noch in den Anfängen. Zunehmend wird jedoch eingefordert, auch jene «Landschaften der Imagination» zu untersuchen, zu der die beobachtenden Wissenschafter selbst gehören (BLAIKIE 1995: 205), und insgesamt den «Rahmungen» (frames) von Natur und Umweltproblemen mehr Beachtung zu schenken (vgl. BRAND u.a. 1997: 9, 27).

\section{Für die Natur sprechen}

Eine interessante und in dieser Hinsicht exemplarische Arbeit aus jüngster Zeit ist WILLEMS-BRAUNs (1997) Aufsatz über die waldbezogenen Konflikte in BritishColumbia. An ihr läßt sich verdeutlichen, welche neuen Perspektiven auf «Natur» eröffnet werden können, wenn solchen «Rahmungen» in der Analyse breiter Raum gegeben wird. Der Autor befaßt sich mit den Auseinandersetzungen über die forstliche Nutzung der alten Waldbestände des Clayoquot Sound an der Westküste von Vancouver Island während der letzten Jahre, und er fragt vor allem danach, warum sich in diesen Auseinandersetzungen bestimmte Positionen Gehör verschaffen können und andere nicht. Der Konflikt sei fast ausschließlich um die polaren Positionen der Umweltgruppen und der Forstindustrie organisiert, während die Stimmen anderer wichtiger Akteure wie der Waldarbeiter, der lokalen Gemeinden und besonders der indigenen Bevölkerung («First Nations») kaum vernehmbar seien. Seine Ausgangsthese besagt, daß bestimmte historische und gegenwärtige Praktiken der Repräsentation die «Natur» als einen leeren Raum ökonomischer und politischer Kalkulation erscheinen lassen und bestimmte Akteure in eine privilegierte Position versetzen, «für» diese Natur zu sprechen (ebd.: 5-7).

WILLEMS-BRAUN entwickelt und stützt diese These vor allem durch die Analyse dreier bildlicher und textlicher Darstellungen, nämlich erstens einer Werbebroschüre der privaten Forstindustrie, zweitens der Reiseberichte des Geologen und Amateurethnologen George Dawson, der die Gegend im Zuge des Geological Survey of Canada um 1880 bereiste, und drittens eines populären Prachtbands über die Region, der 1990 zur Verbreitung der anvisierten Schutzideale vom Western Canada Wilderness Committee herausgegeben wurde. Aus diesen drei Quellen destilliert der Autor sukzessive die Schwierigkeiten der indigenen Bevölkerung, ihre Interessen in dem Konflikt zu artikulieren, was hier nur in Kürze wiedergegeben werden kann: 
Die untersuchten Äußerungen der Forstindustrie konstituieren einen abstrakten Raum und fordern dessen wissenschaftlich-rationale Nutzung. Dabei wird die «normale» Bewirtschaftung des Waldes mit der positiven Entwicklung einer abstrakten Nation verknüpft. $\mathrm{Zu}-$ gleich werden die widerstreitenden sozialen Ansprüche, ob historisch oder aktuell begründet, in einer waldbaulich-technischen Perspektive ausgegrenzt.

Dawsons historische Reiseberichte schließen die indigene Bevölkerung dagegen ein, lösen sie jedoch dabei ganz aus ihrem Kontext. Sie wird zu einem Faktor, der neben den geologischen und forstlichen Ressourcen seinen Platz bekommt, und so trägt Dawson dazu bei, eine duale Vorstellung zu etablieren, wonach es auf der einen Seite eine «Naturraum» gibt, der der aufstrebenden kanadischen Nation als moderner Ressourcenraum zur Verfügung steht, und quasi unabhängig davon einzelne "primitive Orte», nämlich die Dörfer der indigenen Bevölkerung (ebd.: 12-18). Einen spezifischen Sinn entwickelt diese Einteilung erst im Kontext der kolonialen Erschließungspraktiken.

Wie sieht es schließlich auf der naturschützerischen Seite aus? In spektakulären Photographien und halbpoetischen Texten wird auch hier der indigenen Bevölkerung durchaus Raum gegeben. Dabei wird jedoch stets eine enge Harmonie, wenn nicht gar eine Identität des «Traditionellen» mit der Natur suggeriert. Insofern können diese Bevölkerungsgruppen zwar Natur sein, nicht aber aus einer aufgeklärten und modernen Position politisch aktiv für die Natur sprechen. Dies wird durch die Photographien unterstrichen, in denen die Umweltschützer als dynamische und individuelle Akteure erscheinen, während den Indigenen konsequent ein traditionelles Dasein als Teil der «Wildnis» zugeschrieben wird. Somit tragen auch die Umweltgruppen dazu bei, eine differenzierte und zugleich wirkungsmächtige Artikulation der Indigenen in den aktuellen Konflikten zu erschweren (vgl. zum selben Konflikt in anderer Perspektive, aber mit ähnlichem Ergebnis HAYTER \& SOYEZ 1996).

Bei einer solchen Lesart, wie sie hier nur knapp umrissen werden konnte, geht es keineswegs darum, daß die analysierten Rahmungen im gängigen Sinne «falsch» wären. Vielmehr wird herausgearbeitet, daß die verschiedenen Repräsentationen von Natur immer schon bestimmte kategoriale und normative Gehalte mitschleppen und insofern die Konflikte, in denen auf sie zurückgegriffen wird, mit konstituieren und schließlich auch deren aktuelle Verlaufsform beeinflussen.

Durch seinen starken Bezug auf die Modi der Repräsentation von Natur geht WILLEMS-BRAUN über die kulturhistorische Ökologie Sauerscher Prägung hinaus (anderer Ansicht: SLUYTER 1997). Zugleich illustriert dieses Beispiel aber auch einen potentiellen Schwachpunkt der Forschungsansätze, die die gegenwärtigen Naturkonflikte vor allem über eine Analyse von Repräsentationsweisen zu deuten versuchen. So naheliegend und einleuchtend der Einfluß von historischen Diskurslinien und konkreten Bildern auf die Problemdefinitio- nen und Durchsetzungsmöglichkeiten der beteiligten Akteure auch ist, so selten gelingt es wirklich überzeugend, die spezifischen materiellen (sozialen und physischen) Wirkungen dieser Repräsentation herauszuarbeiten. So bleibt bei WILLEMS-BRAUN offen, an welchen konkreten Punkten der Auseinandersetzung die herausgearbeiteten Rahmungen ihre Wirkung entfalten und in welchem Verhältnis ihr Einfluß zu den im engeren Sinne politischen und ökonomischen Prozessen steht. Eine Analyse der Werbekampagnen von Greenpeace, der Diashows von Bruno Manser oder der belletristischen Literatur der Kolonialisten scheint demnach vor allem vielversprechend und produktiv, um die «symbolischen Ressourcen» (BRAND u.a. 1997: 28) auszuloten, die in Naturkonflikten zum Einsatz gebracht werden. Wenn es aber darum geht, wie die verschiedenen Repräsentationen in konkreten Auseinandersetzungen ihre Wirkung entfalten, sind klassische Methoden der Sozialforschung und ökonomische Analysen in ihren Befunden oft überzeugender und ergänzend notwendig, damit den Bildern und Texten nicht ein ähnlich magischer Erklärungswert zugebilligt wird wie in anderen Ansätzen den «objektiven Interessen» oder der systemerhaltenden «Autopoiesis».

\section{Von der konstruierten zur emergenten Natur?}

Die Wirkungsmacht textlicher und bildlicher Repräsentationen ist in der Geographie bisher vor allem dort deutlich gemacht worden, wo es sich um offensichtlich machtdurchdrungene Fragen handelt wie nationale Grenzziehungen oder geopolitische Einteilungen. Wesentlich geringer war bisher das Interesse, auch die Repräsentationen scheinbar natürlicher Gegebenheiten theoretisch zu durchdenken. Erst in jüngster Zeit beginnt etwa eine vorsichtige Debatte über die Implikationen der «simulierten Natur» von Satellitenbildern und Geographischen Informationssystemen (PICKLES 1997), deren Bedeutung in der Definition von Umweltproblemen nicht erst durch die letzten Brände in indonesischen und amazonischen Wäldern sichtbar wurde.

Ein weiterer Grenzbereich von Kultur und Natur, in dem bisher die Humangeographie auffällige Enthaltsamkeit übt, sind die Gen- und Reproduktionstechnologien, die die Idee einer «natürlichen Natur» tief in Frage stellen, welche ja ihre «Prägnanz gerade aus der Entgegensetzung zum Bereich menschlichen Herstellens erhielt» (вÖHME 1994: 115). Dies ist um so erstaunlicher, als es sich um einen Technologiebereich handelt, in dem es von geographischen Metaphern wimmelt («Genomkartierung», "Genorte», «Körperräume» usw.) und der zudem in den klassischen Feldern Industrie und Landwirtschaft Umschichtungen erwarten läßt, die voller «räumlicher» Implikationen stecken. Offensichtlich mischen sich in diesem Bereich die verschiedensten Naturkonstrukte: Während gesellschaftliche Wünsche beginnen materielle Form anzunehmen (die produzierte Natur der Wunderpflanzen und Retortenbabies), entzünden sich an 
Symbolnaturen tiefgreifende Konflikte um Normen und Risiken (die kulturelle Natur der gentechnikfreien Nahrung und der Tierrechte); zugleich scheint sich die erkannte Natur in Information aufzulösen, ja für BAUDRILLARD (1994: 18) ist der genetische Code geradezu das «Idealbild der Virtualität».

Nicht zuletzt dank der Zunahme solcher Mischfelder von Natur und Kultur sind in jüngster Zeit die Grenzziehungen zwischen diesen beiden Großbereichen selbst vermehrt zum Gegenstand der Diskussion geworden. Dabei geht es gerade nicht mehr darum, die Natur von kulturellen Aufladungen zu befreien, oder umgekehrt, die Gesellschaft von natürlichen Verstrickungen freizuhalten, sondern vielmehr diese polaren Grundkategorien selbst als Produkt einer wiederkehrenden Arbeit der «Reinigung» zu analysieren (LATOUR 1995, HARAWAY 1997).

Angesichts der produzierten Natur der gentechnischen Labors und der «dritten Natur» der Body-Shop-Regenwälder könnte die Rede von konstruierten Naturen demnach selbst noch zu sehr in dem Dualismus befangen sein, den sie in Frage zu stellen versucht. Dann wäre es nicht mehr die vorrangige Aufgabe einer kritischen Humangeographie, das Soziale streng abzugrenzen und die Natur als reine «Registrierplatte» menschlicher Aktivität auszusondern bzw. die kulturellen Spuren in dieser Matrix zu lesen (vgl. WERLEN 1997, HARD 1995). Noch weniger kann es allerdings eine Rückkehr zu den alten deterministischen Relationen geben. Am Horizont erscheint vielmehr ein emergentes Phänomen Natur, gleichermaßen «postnatürlich» wie «postsozial», in dessen wiederkehrender Hervorbringung die gesellschaftlichen Konstrukte ebenso wie die natürliche Materialität im doppelten Wortsinn aufgehoben wären. Die theoretische und methodologische Herausforderung besteht dann vor allem darin zu zeigen, wie sich Materialität und Repräsentation auch im wissenschaftlichen Zugriff verknüpfen lassen, ohne in beliebigen «sozialen Texten» zu enden. Für die Geographie, deren «Brückenproblem» oft genug benannt wurde, beinhaltet dies nicht das Versprechen einer wiederhergestellten Einheit, jedoch vielfältige Perspektiven der Kooperation innerund außerhalb der Disziplin im Blick auf die Vielfalt der sozial-natürlichen Emergenzen in einem globalen «Konfliktfeld Natur» (vgl. FLITNER u.a. 1998).

\section{Literatur}

BAHRENBERG, G. (1994): Geographie und Humanökologie. In: ERNSTE, H. (Hg.), Pathways to Human Ecology: From Observation to Commitment, Bern, 57-68.

BAUDRILLARD, J. (1978): Die Agonie des Realen, Berlin. BAUDRILLARD, J. (1994): Die lllusion und die Virtualität, Bern. BERGER, P., \& LUCKMANN, T. L. (1969): Die gesellschaftliche Konstruktion der Wirklichkeit, Frankfurt am Main.

BLAIKIE, P. (1995): Changing Environments or Changing Views? Geography Vol. 80, 203-214.
BÖHME, G. (1992): Natürlich Natur. Über Natur im Zeitalter ihrer technischen Reproduzierbarkeit, Frankfurt am Main.

BRAND, K.W., EDER, K., POFERL, A. (1997): Ökologische Kommunikation in Deutschland, Opladen.

BRYANT, R. L., \& BAILEY, S. (1997): Third World Political Ecology, London \& New York.

BUTLER, J. (1995): Körper von Gewicht: die diskursiven Grenzen des Geschlechts, Berlin.

CASTREE, N. (1995): The Nature of Produced Nature: Materiality and Knowledge Construction in Marxism. Antipode Vol. 27, 1, 12-48.

CASTREE, N., \& BRAUN, B. (1998): The construction of nature and the nature of construction: analytical tools for building survivable futures. In: Dies. (Hg.): Remaking Reality: Nature at the Millenium, London, i.E.

CRONON, W. (1995): The Trouble with Wilderness or Getting Back to the Wrong Nature. In: Ders. $(\mathrm{Hg}$.): Uncommon Ground: Toward Reinventing Nature, New York \& London, 69-90.

DESCOLA, Ph. (1996): Constructing Natures: Symbolic Ecology and Social Practice. In: DESCOLA, PH., \& PÁLSSON, G. ( $\mathrm{Hg}$.): Nature and Society. Anthropological Perspectives, London \& New York, 82-102.

ECO, U. (1987): Orwellsche Ökologie und fleischgewordene Coca-Cola. In DERS.: Über Gott und die Welt, München, 89-99.

EDER, K. (1988): Die Vergesellschaftung der Natur. Studien zur sozialen Evolution der praktischen Vernunft, Frankfurt am Main.

ELLEN, R., \& FUKUI, K. (Hg.) (1996): Redefining Nature: Ecology, Culture and Domestication, Oxford/Washington.

ESCOBAR, A. (1996): Constructing Nature: Elements for a Poststructural Political Ecology. In: PEET, R., \& WATTS, M. $(\mathrm{Hg}$.$) : Liberation Ecologies, London \& New York, 46-68.$

FITZSIMMONS, M. (1989): The Matter of Nature. Antipode Vol. 21, No. 2, 106-120.

FLITNER, M., GÖRG, C., \& HEINS, V. (Hg.) (1998): Konfliktfeld Natur: Biologische Ressourcen und globale Politik, Opladen, i.E.

GERBER, J. (1997): Beyond Dualism - the Social Construction of Nature and the Natural and Social Construction of Human Beings. Progress in Human Geography Vol. 21, 1, $1-17$.

GÖRG, C. (1998): Die Regulation der biologischen Vielfalt und die Krise gesellschaftlicher Naturverhältnisse. In: FLITNER u. a. (Hg.) (1998): Konfliktfeld Natur: Biologische Ressourcen und globale Politik, Opladen, i.E.

HARAWAY, D. (1992): The Promises of Monsters: A Regenerative Politics for Inappropriate/d Others. In: GROSSBERG, L., u.a. (Hg.): Cultural Studies, New York / London, 295-337.

HARAWAY, D. (1997): Modest_Witness@Second_Millenium: FemaleMan ${ }^{\odot}$ _Meets_OncoMouse ${ }^{\mathrm{TM}}$, New York / London. HARD, G. (1983): Zu Begriff und Geschichte der "Natur" in der Geographie. In: GROSSKLAUS, G., \& OLDEMEYER, E. (Hg.): Natur als Gegenwelt: Beiträge zur Kulturgeschichte der Natur, Karlsruhe, 139-167.

HARD, G. (1995): Spuren und Spurenleser: Zur Theorie und Ästhetik des Spurenlesens in der Vegetation und anderswo, Osnabrück.

HARVEY, D. (1996): Justice, Nature and the Geography of Difference, Oxford.

HAYLES, N.K. (1995): Simulated Natures and Natural Simulations: Rethinking the Relationship between the Beholder and the World. In: CRONON, W. (Hg.): Uncommon Ground: Toward Reinventing Nature, New York \& London, 409-425. HAYTER, R., \& SOYEZ, D. (1996): Clearcut Issues: German Environmental Pressure and the British Columbia Forest Sector. GZ, 3-4, 143-156. 
HECHT, S., \& COCKBURN, A. (1989): The Fate of the Forest: Developers, Destroyers and Defenders of the Amazon, London.

KNORR CETINA, K. (1981): The Manufacture of Knowledge. An Essay on the Constructivist and Contextual Nature of Science, Oxford.

KNORR CETINA, K. (1997): Sociality of Objects: Social Relations in Postsocial Knowledge Societies. Theory, Culture \& Society 14 (4), 1-30.

LATOUR, B. (1995): Wir sind nie modern gewesen, Berlin.

LATOUR, B., \& WOOLGAR, S. (1979): Laboratory Life: The Social Construction of Scientific Facts, Beverly Hills.

MATURANA, H., \& VARELA, F. (1987): Der Baum der Erkenntnis. Die biologischen Wurzeln des menschlichen Handelns, Bern.

MCKIBBEN, B. (1989): The End of Nature, New York.

MOORE, D. (1993): Contesting terrain in Zimabwe's Eastern Highlands: political ecology, ethnography, and peasant resource struggles. Economic Geography Vol. 69, 380-401. MOSCOVICI, S. (1982): Versuch über die menschliche Geschichte der Natur, Frankfurt.

OLWIG, K.R. (1996): Nature: Mapping the Ghostly Traces of a Concept. In: EARL, C., MATHEWSON, K., \& KENZER, M.S. (Hg.): Concepts in Human Geography, London, 63-96.

PEET, R., \& WATTS, M. (Hg.) (1996): Liberation Ecologies: Environment, Development, Social Movements, London/ New York.

PELUSO, N. (1992): Rich Forests, Poor People: Resource Control and Resistance in Java, Berkeley.

PICKLES, J. (1997): Tool or Science? GIS, Technoscience and the Theoretical Turn. Annals of the Association of American Geographers Vol. 87, 2, 363-372.

SLUYTER, A. (1997): On Excavating and Burying Epistemologies (Commentary on Willems-Braun's "Buried Epistemol- ogies"). Annals of the Association of American Geographers Vol. 87, 4, 700-702.

SMITH, N. (1984): Uneven Development: Nature, Capital and the Production of Space, Oxford.

SOKAL, A. (1996): A Physicist's Experiments with Cultural Studies. Lingua Franca 6, 62-64.

SPIVAK, G. (1996): The Spivak Reader (ed. by D. Landry \& G. McLean). New York/London.

THOMPSON, M., ELLIS, R. \& WILDAVSKY, A. (1990): Cultural Theory or Why all that is permanent is bias, Boulder.

VAN DEN DAELE, W. (1996): Soziologische Beobachtung und ökologische Krise. Kölner Zeitschrift für Soziologie und Sozialpsychologie, SoH 36/1997, 420-440.

WARK, M. (1994): Third Nature. Cultural Studies 8, 1994, 115-132.

WARNKE, M. (1992): Politische Landschaft: zur Kunstgeschichte der Natur, München.

WATTS, M. (1989): The Agrarian Question in Africa. Progress in Human Geography Vol. 13, 1-41.

WELSCH, W. (1993): Unsere Postmoderne Moderne, Berlin. (4. Auflage)

WERLEN, B. (1997): Sozialgeographie alltäglicher Regionalisierungen. Band 2: Region, Regionalisierung und Globalisierung, Stuttgart.

WILLEMS-BRAUN, B. (1997): Buried Epistemologies: The Politics of Nature in (Post)colonial British Columbia. Annals of the Association of American Geographers Vol. 87, 1, 3-31.

WILLIAMS, M. (1994): The Relation of Environmental History and Historical Geography. Journal of Historical Geography Vol. 20, 1, 3-21.

ZIMMERER, K. S. (1996): Ecology as Cornerstone and Chimera in Human Geography. In: EARL, C., MATHEWSON, K., \& KENZER, M.S. (Hg.): Concepts in Human Geography, London, 161-188. 